\title{
LIFT: a focal-plane wavefront sensor for real-time low-order sensing on faint sources
}

\author{
Serge Meimon,* Thierry Fusco, and Laurent M. Mugnier \\ Office National d'Études et de Recherches Aérospatiales, Département d’Optique Théorique et Appliquée, \\ BP 72, F-92322 Châtillon Cedex, France \\ ${ }^{*}$ Corresponding author: meimon@onera.fr
}

Received June 22, 2010; revised July 29, 2010; accepted August 5, 2010;

posted August 16, 2010 (Doc. ID 126599); published September 3, 2010

We propose the linearized focal-plane technique (LIFT) and compare it to classical sensors, such as the quad-cell wavefront sensor (WFS), pyramid WFS, and Shack-Hartmann WFS. The number of modes sensed by LIFT can be tuned without any hardware modification nor degradation of low-order sensing performance. We derive an analytic model of the noise propagation law, which we validate on end-to-end simulations. (c) 2010 Optical Society of America

OCIS codes: $\quad 010.1080,010.7350$.

Laser-assisted adaptive optics (AO) systems are opening a new era in ground-based high-resolution imaging and spectroscopy. They should be able to increase dramatically the system sky coverage, thus solving the main limitation of current AO systems. Unfortunately, the laser guide star (LGS) wavefront sensing (WFS) principle is insensitive to tip/tilt, and focus measurement is corrupted by the evolution of the sodium concentration in altitude. Hence, low-order modes have to be removed from the LGS WFS signal and measured separately using faint natural guide stars (NGSs). In essence, the final system sky coverage is dictated by the signal-to-noise ratio (SNR) of the low-order NGS measurements. In that framework, we propose a new focal-plane WFS concept called the linearized focal-plane technique (LIFT), which allows us to efficiently deal with low-order mode measurement under low-flux conditions.

The most common way to sense tip/tilt is to form a focal plane image and detect its position with regard to a reference position. Quad-cell (QC) and pyramid WFSs [1] split the focal-plane image in four zones and extract the spot position from the photometric balance between the four zones. To sense only tip/tilt, one pixel for each of the four facets is enough for the pyramid, making it analogous to QC (yet heavier in terms of optical design and sometimes difficult to implement [2]). Another way is to use a finer grid and to compute the position by means of a center of gravity (CoG). In this case, more detector pixels are used, so a weighted center of gravity (WCoG) can be used to reduce detector noise [3].

When one wants to sense focus in addition to tip/tilt, the hardware realization of these sensors has to be modified. QC and CoG/WCoG can be used for each lenslet of a $2 \times 2$ subaperture Shack-Hartmann (SH) WFS. This adaptation corresponds to an additional splitting in the pupil plane, resulting in a strong increase of photon noise variance $(\times 4)$ and detector noise variance $(\times 16)$ on tip/tilt measurement. With regard to the pyramid WFS, no additional pupil plane splitting is needed, so there is no photon noise increase. Still, there will be detector noise increase, and the hardware has to be modified to extend the detector matrix.

An alternative solution is to build a modified quad cell (MQC) able to sense tip, tilt, and focus, with the same tip/tilt sensing capabilities as a classical QC. A classical $\mathrm{QC}$ allows, by applying a projector $P_{\mathrm{QC}}$ to the four pixel values, to estimate four modes, which are total flux, tip, tilt, and astigmatism, respectively:

$$
\begin{aligned}
\text { flux : } & P_{\mathrm{QC}}=\left[\begin{array}{ll}
1 & 1 \\
1 & 1
\end{array}\right], & \text { tip : } & P_{\mathrm{QC}}=\left[\begin{array}{cc}
1 & -1 \\
1 & -1
\end{array}\right], \\
\text { tilt : } & P_{\mathrm{QC}}=\left[\begin{array}{cc}
-1 & -1 \\
1 & 1
\end{array}\right], & \text { astig. : } & P_{\mathrm{QC}}=\left[\begin{array}{cc}
1 & -1 \\
-1 & 1
\end{array}\right],
\end{aligned}
$$

by subtracting the diagonals. Adding a small astigmatism allows us to "code" defocus on the fourth mode: at the best focus, the image has an $\mathrm{X}$ shape, whereas with a defocus, the image stretches in one diagonal direction or the other, whether the focus is positive or negative (see Fig. 1). Such a MQC was envisioned at Keck Observatory in the 1990s [4]. It was, however, discarded because of its two fundamental limitations: (i) the linearity range is limited and (ii) higher modes are aliased, as discussed in the following.

The second limitation is critical, as there is no way to discriminate between defocus and astigmatism, nor between defocus and sphere (see Fig. 1). To solve this limitation, we investigated the possibility of using a finer sampling (e.g., Shannon sampling) instead of only four quadrants. In the following section, we describe the LIFT.

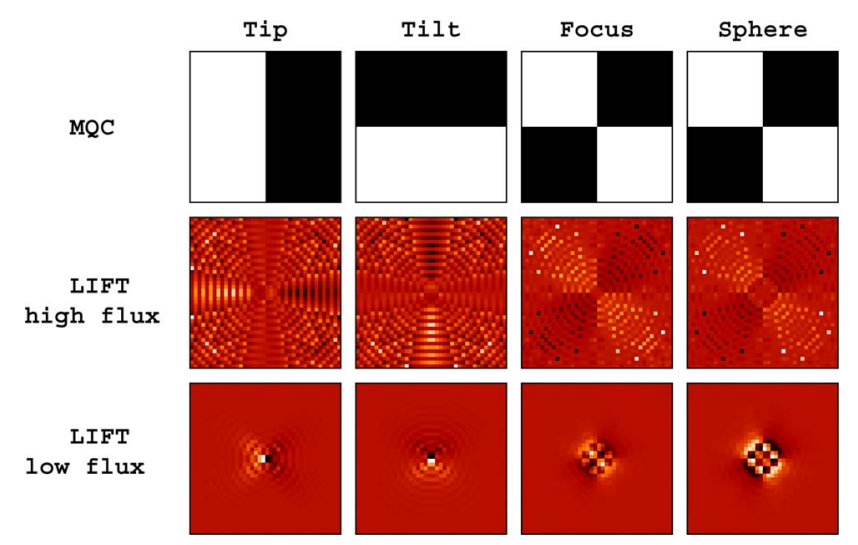

Fig. 1. (Color online) Weighting modes for the MQC and LIFT (in high- and low-flux cases). 
The LIFT is a linearized focal-plane sensor, using a $\pi / 8$ radian astigmatism offset $\phi_{d}$. This offset solves the classical phase undetermination problem for even modes [5]. The first-order approximation of the phase retrieval problem was described by Gonsalves [6] . Let $\boldsymbol{r}$ (or $\boldsymbol{u}$ ) be the spatial coordinates in the focal (or pupil) plane, $\phi$ the phase to sense and $n$ the noise on the image. We assume a point-source object. The image formed at the focal plane is given by

$$
I(\boldsymbol{r})=\left|\mathrm{FT}\{\underbrace{P e^{i \phi_{d}(\boldsymbol{u})}}_{P_{d}} \cdot e^{i \phi}\}\right|^{2}+n(\boldsymbol{r}),
$$

where FT is a Fourier transform (data images are normalized to a unitary flux). Let us denote $\boldsymbol{A}$ as the vector of coefficients $a_{i}$ corresponding to a modal decomposition of the phase to sense, e.g., the Zernike polynomials $Z_{i}$ [7]. The first-order Taylor expansion with regard to $A$ gives

$$
I(\boldsymbol{A})-I(\mathbf{0}) \simeq \sum_{k} a_{k} \cdot I_{k}^{\prime}+n,
$$

with $I_{k}^{\prime}=\frac{\partial I(\boldsymbol{A})}{\partial a_{k}}=2 \Re\left\{\tilde{P}_{d}^{*} \cdot i \mathrm{FT}\left\{P_{d} \cdot Z_{k}\right\}\right\}$. We assume that the image is formed on a matrix of pixels. For the $j$ th pixel, we define $\Delta I[j] \triangleq I(\boldsymbol{A})[j]-I(\mathbf{0})[j]$ and $H[j, k] \triangleq I_{k}^{\prime}[j]$. Equation (1) can be recast in a standard linear inverse problem:

$$
\Delta \boldsymbol{I}=\boldsymbol{H} \cdot \boldsymbol{A}+\boldsymbol{n}
$$

We assume that $\boldsymbol{n}$ is a zero-mean Gaussian noise, with a covariance matrix $\boldsymbol{R}_{n}$. The maximum likelihood estimator is given by

$$
\hat{\boldsymbol{A}}_{\mathrm{ML}}=P_{\mathrm{ML}} \Delta \boldsymbol{I} ; \quad P_{\mathrm{ML}}=\left(\boldsymbol{H}^{t} \boldsymbol{R}_{\boldsymbol{n}}^{-\mathbf{1}} \boldsymbol{H}\right)^{-1} \boldsymbol{H}^{t} \boldsymbol{R}_{\boldsymbol{n}}^{-\mathbf{1}} .
$$

The spatial variance of the estimation error $\boldsymbol{E} \triangleq \boldsymbol{A}-\hat{\boldsymbol{A}}_{\mathrm{ML}}$ is given by

$$
\operatorname{var}=\operatorname{Tr}\left\{\left\langle\boldsymbol{E} \boldsymbol{E}^{t}\right\rangle\right\}=\operatorname{Tr}\left\{\left(\boldsymbol{H}^{t} \boldsymbol{R}_{\boldsymbol{n}}^{-\mathbf{1}} \boldsymbol{H}\right)^{-1}\right\} .
$$

The level of the astigmatism offset $\phi_{d}$ has to be set according to the level of aberrations to solve. Our method is intended to work in a good correction case (Strehl ratio $\geq 30 \%$ ), i.e., in a closed loop [8] or after a first correction stage (e.g., in a multiconjugate $\mathrm{AO}$ system). We can, therefore, consider a small offset, which ensures a high SNR. The $\pi / 8$ radian offset value we selected was obtained with a rule of thumb and has not been optimized yet. In particular, it could be updated online, depending on the strength of the aberration to sense.

The equivalent of the weighing modes $P_{\mathrm{QC}}$ is found in the columns of $P_{\mathrm{ML}}$ [see Eq. (3)]. We have illustrated the fact that the $P_{\mathrm{QC}}$ modes are binned versions of the $P_{\mathrm{ML}}$ modes in Fig. 1. We see that, depending on the noise statistics specified to the algorithm (via the $\boldsymbol{R}_{n}^{-1}$ matrix), the LIFT automatically adjusts the adequate number of pixels needed. For instance, in a low-flux regime, only a few central pixels are used in the estimation in order to minimize detector-noise propagation.

Unlike the MQC, for which spherical aberration has exactly the same signature as focus, the LIFT allows us to sense higher-order modes. More generally, as regards aliasing limitations, the LIFT performs better than MQC for two reasons: (i) the LIFT sampling allows us to sense other orders than the mere tip, tilt and focus, without any modification of the device. The maximum number of modes that can be sensed is limited by the number of pixels involved in the estimation, as well as the level of noise. At this stage, the optimization of the number of sensed mode is still an open issue; (ii) by limiting the number of pixels, we hereby limit the field. This is the equivalent of a pinhole filtering method proposed for SH [9] for aliasing mitigation purposes.

Fundamentally, the MQC and LIFT have close linearity domains, as they differ only in the way they weight photons. However, the limited expansion of Eq. (1) can be performed around the current estimate of the aberrations $\hat{\boldsymbol{A}}$ so that the residual error is estimated:

$$
I(\boldsymbol{A})-I(\hat{\boldsymbol{A}}) \simeq \sum_{k} \delta a_{k} \cdot I_{k}^{\prime}+n .
$$

The obtained incremental values $\delta a_{k}$ are added to the current estimate of the Zernike coefficients of the phase to sense. This process is cycled three times (more iterations do not yield a major improvement). This helps in increasing the LIFT linearity range, making it much larger than that of the MQC (cf. Fig. 2). The LIFT is therefore unbiased over the whole linearity range.

A by-product is that the LIFT in the undersampled Shannon/2 sampling version (LIFT S/2) is also unbiased over the whole linearity range. Unlike the undersampled SH case, no gain correction has to be applied, provided that the image formation model of Eq. (1) takes into account the correct sampling.

In what follows, we derive noise propagation laws for the LIFT and compare it with the MQC and SH with CoG or WCoG centroiding.

Classical WFSs have similar behavior in terms of photon and detector noises [10]:

$$
\sum_{i} \sigma^{2}\left(\hat{a}_{i}-a_{i}\right)=\sum_{i} \alpha_{i} \frac{1}{n_{\mathrm{ph}}^{\text {tot }}}+\sum_{i} \beta_{i}\left(\frac{\sigma_{e}}{n_{\mathrm{ph}}^{\text {tot }}}\right)^{2},
$$

where $n_{\mathrm{ph}}^{\text {tot }}$ is the total number of photons at the entrance of the WFS and $\sigma_{e}$ is the rms number of noise electrons per pixel and per frame.

The MQC coefficients $\alpha_{i}^{\mathrm{MQC}}, \beta_{j}^{\mathrm{MQC}}$ were obtained via a diffractive simulation (the values $\alpha_{2}^{\mathrm{MQC}}$ and $\beta_{2}^{\mathrm{MQC}}$ we obtained are consistent with analytic formulas for classical
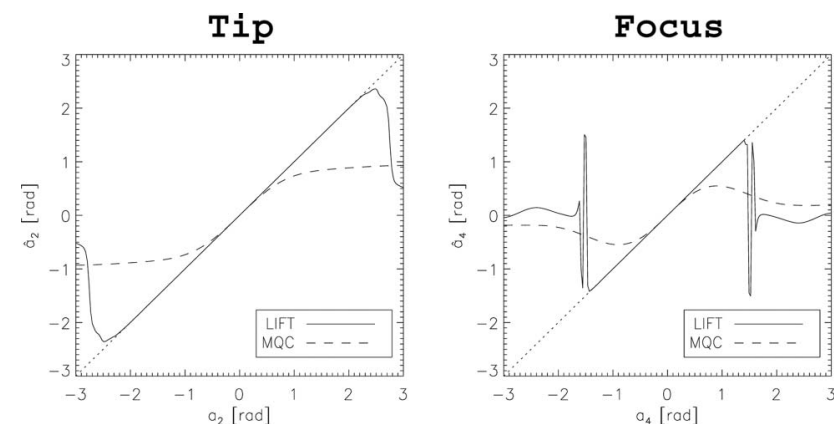

Fig. 2. Linearity ranges for the LIFT and MQC in tip and focus. 
Table 1. $\sum \alpha_{i} / \sum \beta_{i}$ : Total Photon/Detector Noise Variance in $\operatorname{rad}^{2}$ for $n_{\mathrm{ph}}^{\text {tot }}=1 \mathrm{ph} . e^{-}, \sigma_{e}=1 \mathrm{ph} . e^{-}$

\begin{tabular}{|c|c|c|c|c|c|}
\hline Sensed Modes & $Z_{2}, Z_{3}$ & $Z_{2}, Z_{3}, Z_{4}$ & $N \leq 2$ & $N \leq 3$ & $N \leq 4$ \\
\hline MQC & $1.48 / 6$ & $3.52 / 14$ & - & - & - \\
\hline $\mathrm{SH}$ (CoG) & $1.01 / 1920$ & $4.61 />10^{4}$ & $6.38 />10^{4}$ & $17.94 />10^{4}$ & $36.76 />10^{4}$ \\
\hline SH (WCoG) & $1.80 / 18$ & $8.19 / 334$ & $11.33 / 462$ & $31.90 / 2928$ & $65.34 />10^{4}$ \\
\hline LIFT & $0.84 / 26$ & $1.39 / 105$ & $2.44 / 363$ & $4.11 / 1044$ & $8.48 / 8252$ \\
\hline LIFT S/2 $2^{a}$ & $0.84 / 7$ & $1.44 / 35$ & $2.72 / 139$ & $4.99 / 544$ & $10.59 / 4526$ \\
\hline
\end{tabular}

${ }^{a} \mathrm{~S} / 2$ corresponds to a Shannon/2 sampling.

QC tip/tilt error [10] with less than $6 \%$ discrepancy). Regarding $\mathrm{SH}$, we used centroid error formulas derived by Nicolle et al. [3] for CoG and WCoG (assuming a Shannon sampling, diffraction-limited spot size, and $3 \times 3$ pixel zone used for centroid estimation; the propagation of this error on Zernike modes is obtained via the RigautGendron coefficients [11]). We considered an $N \times N$ subaperture geometry, with $N$ being the maximum radial order of the modes to sense. The number of photons per subaperture scales in $n_{\mathrm{ph}}^{\text {tot }} / N^{2}$, so $\alpha_{i}^{\mathrm{SH}}$ and $\beta_{i}^{\mathrm{SH}}$ scale, respectively, in $N^{2}$ and $N^{4}$. LIFT analytical coefficients $\alpha_{i}^{\mathrm{LIFT}}, \beta_{i}^{\mathrm{LIFT}}$ are derived from Eq. (4) (the diagonal components of $\left\langle\boldsymbol{E} \boldsymbol{E}^{t}\right\rangle$ are the phase-error variance for each mode).

The $\sum \alpha_{i}$ and $\sum \beta_{i}$ values [cf. Eq. ( $\left.\underline{6}\right)$ ] obtained for an increasing number of sensed modes are shown, respectively, in Table 1. The LIFT yields the lowest photon noise, whatever the sampling factor. The gain in photon noise over a SH with CoG centroiding tends to a factor of 10 for a high number of modes. In the detector-noise regime, the most efficient sensor for tip, tilt, and focus is the MQC. However, as soon as astigmatism or higher modes are present, the MQC has to be discarded. The LIFT is more efficient in terms of detector noise than a SH with WCoG as soon as the astigmatisms have to be sensed in addition to tip, tilt, and focus. In a Shannon/2 configuration, the LIFT yields comparable performance as the MQC on tip, tilt, and focus sensing. This, in addition to the fact that the LIFT can be tuned to estimate the desired number of modes without hardware modification nor low-order performance degradation, make it an attractive solution for few-order sensing.

We have proposed the LIFT, which allows us to efficiently deal with low-order mode measurements. This sensor allows us to estimate several modes from a full pupil image. Because of the full aperture gain, it favorably compares to other WFSs in terms of noise propaga- tion, hardware simplicity, and on-site tunability (it can be adapted to the perturbation level and to the NGS magnitude).

An end-to-end simulation of the locking regime for tip, tilt, and focus was performed in the framework of the ATLAS study [8], which concluded in a successful use of the LIFT in a good correction case. Of course, the next step is the experimental validation, first on a dedicated bench and, eventually, on the sky.

The authors are thankful to Richard Dekany for fruitful discussions about early testing of the MQC at Keck Observatory and to Frederic Cassaing for sharing his intuitions. This study was supported in part by the European Southern Observatory.

\section{References and Notes}

1. R. Ragazzoni and J. Farinato, Astron. Astrophys. 350, L23 (1999).

2. B. Neichel, F. J. Rigaut, M. Bec, D. Gratadour, M. Bocca, and G. Trancho, Proc. SPIE 7736, 773606 (2010).

3. M. Nicolle, T. Fusco, G. Rousset, and V. Michau, Opt. Lett. 29, 2743 (2004).

4. Private conversation with R. Dekany, Caltech Optical Observatories, Physics, Math and Astronomy Division, California Institute of Technology, Pasadena, Calif. 91125, USA.

5. A. Tokovinin and S. Heathcote, Pub. Astron. Soc. Pacific 118, 1165 (2006).

6. R. A. Gonsalves, Opt. Lett. 26, 684 (2001).

7. R. J. Noll, J. Opt. Soc. Am. 66, 207 (1976).

8. T. Fusco, S. Meimon, Y. Clenet, M. Cohen, J. Paufique, and H. Schnetler, Proc. SPIE 7736 77360D (2010).

9. L. A. Poyneer and B. Macintosh, J. Opt. Soc. Am. A 21, 810 (2004).

10. G. Rousset, in Adaptive Optics in Astronomy, F. Roddier, ed. (Cambridge U. Press, 1999), Chap. 5, pp. 91-130.

11. F. Rigaut and E. Gendron, Astron. Astrophys. 261, 677 (1992). 